\title{
Infrared spectroscopic studies of hydrogenated silicon clusters
}

\section{Guiding the search for $\mathrm{Si}_{2} \mathrm{H}_{\mathrm{x}}$ species in the Circumstellar Envelope of IRC+10216}

\author{
R. I. Kaiser ${ }^{1,2}$ and Y. Osamura ${ }^{3}$ \\ ${ }^{1}$ Department of Chemistry, University of Hawai'i at Manoa, Honolulu, HI 96822, USA \\ e-mail: kaiser@gold.chem.hawaii.edu \\ 2 Department of Physics and Astronomy, The Open University, Milton Keynes MK7 6AA, UK \\ 3 Department of Chemistry, Rikkyo University, 3-34-1 Nishi-ikebukuro, Tokyo 171-8501, Japan
}

Received 19 February 2004 / Accepted 8 November 2004

\begin{abstract}
Silicon-bearing species $\mathrm{Si}_{2} \mathrm{H}_{x}(x=1-6)$ are probable candidates in the circumstellar envelope of IRC+10216. We have observed several fundamentals of new silicon-containing radicals $\mathrm{Si}_{2} \mathrm{H}_{3}$ and $\mathrm{Si}_{2} \mathrm{H}_{5}$ in addition to the well-known $\mathrm{Si}_{2} \mathrm{H}_{4}$ and $\mathrm{Si}_{2} \mathrm{H}_{6}$ species from infrared spectroscopy in low temperature silane matrices at $10 \mathrm{~K}$. Several infrared bands identify the $\mathrm{Si}_{2} \mathrm{H}_{x}$ species and can be used to search for these molecules in the circumstellar envelope of IRC +10216 . These infrared bands are confirmed by ab initio quantum chemical calculation as well as via corresponding infrared spectra detected for the deuterated species $\mathrm{Si}_{2} \mathrm{D}_{x}$.
\end{abstract}

Key words. molecular data - molecular processes - astrochemistry

\section{Introduction}

Since the pioneering detection of the silane molecule $\left(\mathrm{SiH}_{4}\right)$ in the circumstellar envelope of the carbon star IRC+10216 (CW Leo) (Barratt 1978; Goldhaber \& Betz 1984), astronomers identified nine silicon-bearing molecules in the gas phase of cold molecular clouds, star-forming regions, and circumstellar envelopes of evolved carbons stars. These individual molecules can be arranged in four groups: simple diatomic molecules of the general chemical formula SiX (group 1), silicon carbides holding the generic composition $\mathrm{SiC}_{n}$ (group 2), and silicon cyanides (group 3). The diatomic species silicon carbide ( $\mathrm{SiC})$, silicon sulfide (SiS), and silicon nitride $(\mathrm{SiN})$ are important constituents of the circumstellar envelope of CW Leo (Cernicharo et al. 1989; Speck et al. 1997; Bieging \& Nguyen 1989; Turner 1992). The second group - the silicon carbides contains three members, i.e. the cyclic, $\mathrm{C}_{2 \mathrm{v}}$ symmetric silicon dicarbide molecule, $\mathrm{SiC}_{2}$ (Thaddeus et al. 1984; Sarre et al. 1996; Chandra \& Sahu 1993; Gensheimer \& Snyder 1997; Lloyd Evans et al. 2000), a polar, rhomboidal bicyclic $\mathrm{SiC}_{3}$ species (McCarthy et al. 1999; Apponi et al. 1999), and a closed-shell linear chain molecule of the formula $\mathrm{SiC}_{4}$ (Ohishi et al. 1989). Silicon carbide and silicon dicarbide have similar column densities, whereas the higher members silicon tricarbide $\left(\mathrm{SiC}_{3}\right)$ and silicon tetracarbide $\left(\mathrm{SiC}_{4}\right)$ are 200 times less abundant, with silicon tricarbide $50 \%$ more copious than silicon tetracarbide. The last group contains only one molecule, i.e. the linear $\mathrm{SiCN}$ species (silicon monocyanide), which has also been detected in the envelope of this carbon star (Guelin et al. 2000). It is important to stress that some molecules like SiS were also detected in vibrationally excited $(v=1)$ states (Turner 1987). Note that silicon monoxide (SiO) has only been observed in star forming regions such as Sagittarius B2 (Peng et al. 1995; Schilke et al. 1997) and to a lesser extent in cold molecular clouds (Ziurys et al. 1989), but not in circumstellar envelopes of carbon-rich stars; a possible formation route of silicon monoxide might involve barrier-less reactions with molecular oxygen and the hydroxyl radical (LePicard et al. 2001).

Silicon, which is - besides helium and neon - the fifth most abundant element in the interstellar medium with abundances of $3 \times 10^{-5}$ relative to hydrogen, does not exist only in isolated gas phase molecules, but also as silicon-bearing nano particles - so-called dust grains - in circumstellar envelopes of latetype stars. As evident from the $11 \mu \mathrm{m}$ emission feature, silicon subsists as silicon carbide nano particles in carbon-rich giants (Goebel et al. 1995; Blanco et al. 1998). Pillinger \& Russel (1993) identified these silicon carbide particles also in meteorites; their role as cometary dust particles should be pointed out, too (Orofino et al. 1994). If the central star is oxygen-rich, silicates such as olivine $\left((\mathrm{Fe}, \mathrm{Mg})_{2} \mathrm{SiO}_{4}\right)$ dominate their spectra (Fabian et al. 2001). Note that crystalline silicon nanoparticles might also explain the red emission as observed, for example, in the Red Rectangle (Ledoux et al. 2001). The assignment of silicon disulfide $\left(\mathrm{SiS}_{2}\right)$ as a carrier of the unidentified $21 \mu \mathrm{m}$ 
emission feature must be regarded as tentatively (Kraus et al. 1997).

The abundances of these highly unsaturated, siliconbearing molecules were first discussed in terms of $\mathrm{Si}^{+}\left({ }^{2} \mathrm{P}_{j}\right)$-initiated ion-molecule reactions (Glassgold et al. 1986). This model has been expanded later by photon-induced reactions and photodissociation processes to account for the observation of the newly discovered $\mathrm{SiC}_{3}$ and $\mathrm{SiC}_{4}$ species (Glassgold \& Mamon 1992; Howe \& Millar 1994; Willacy \& Cherchneff 1998) and for the abundances of silicon monoxide in interstellar clouds and in photon-dominated regions (Langer \& Glassgold 1990; Walmsley et al. 1999). Also, Mackay (1995) investigated the chemistry of silicon in hot molecular cores, identifying silane as a reservoir for silicon. However, with the exception of silane, no hydrogenated, silicon-containing molecule has ever been observed in the interstellar medium.

From the astrochemical viewpoint, this is quite surprising. Recent laboratory experiments investigated the photochemistry of silane and the subsequent reactions of the photodissociation products. These data suggested that more complex, hydrogenated silicon clusters of the generic formula $\mathrm{Si}_{2} \mathrm{H}_{x}(x=1-6)$ are formed in these processes (Cook et al. 2001; Zavelovich \& Lyman 1989; Hu et al. 2003; Glenewinkel-Meyer et al. 1993; Tonokura et al. 1992; Perkins et al. 1979; Borsella et al. 1988; Ashfold et al. 1996; Aka \& Boch 2002; Wang \& Huang 1998; Oikawa et al. 1994). Their experiments suggest that a rich silane chemistry is expected in the circumstellar envelope of IRC +10216 : photochemistry of silane molecules close to the photosphere could contribute to the formation of small hydrogenated silicon clusters of the generic formula $\mathrm{Si}_{2} \mathrm{H}_{x}$. One drawback of astronomical observations are their vanishing $\left(\mathrm{Si}_{2} \mathrm{H}_{6}\right)$ or relatively small dipole moments of less than 1.1 Debye. Therefore, an infrared spectroscopic search of the $\mathrm{Si}_{2} \mathrm{H}_{x}$ species might present a viable alternative. To guide a search for hydrogenated silicon clusters in the circumstellar envelope of IRC +10216 , we present in this paper a combined experimental and theoretical investigation of the infrared spectra and the vibronic levels together with their intensities of various $\mathrm{Si}_{2} \mathrm{H}_{x}(x=1-6)$ isomers. These data will assist a positive identification of hydrogen-rich silicon clusters in future infrared spectroscopic surveys of the circumstellar envelope of IRC +10216 .

\section{Computational approach}

The molecular structures and vibrational frequencies of the $\mathrm{Si}_{2} \mathrm{H}_{x}(x=1-6)$ isomers and their fully deuterated counterparts were scrutinized in terms of ab initio molecular orbital methods. The geometries were optimized with the hybrid density functional method (B3LYP), i.e. Becke's threeparameter non-local exchange functional (Becke 1993) with the non-local correlation functional of Lee et al. (1988) and the $6-311 \mathrm{G}(\mathrm{d}, \mathrm{p})$ basis set (Krishnan et al. 1980). Since the energies of the quartet electronic states of the $\mathrm{Si}_{2} \mathrm{H}_{x}(x=$ $1,3,5)$ species and the triplet electronic states of the $\mathrm{Si}_{2} \mathrm{H}_{x}$ $(x=4,6)$ isomers are extremely high, we have only computed the doublet and singlet electronic states. To facilitate spectroscopic identification in circumstellar envelopes, we also obtained vibrational frequencies and infrared intensities. The coupled cluster CCSD(T) calculations (Cizek 1969; Pople et al. 1987) with the aug-cc-pVTZ basis set (Kendall et al. 1992) were also performed at the optimized structures obtained with the B3LYP method. All computations were carried out using the GAUSSIAN 98 program package (Frisch et al. 2002). The relative energies stated in the text are the values obtained with the $\operatorname{CCSD}(\mathrm{T})$ method corrected with the zero-point vibrational energies obtained with the B3LYP method.

\section{Experimental procedure}

Our experiments were conducted in a contamination-free ultrahigh vacuum (UHV) chamber which can be evacuated down to $2 \times 10^{-10}$ torr by a magnetically suspended turbopump backed by an oil-free scroll pump. A rotatable, two-stage closed-cycle helium refrigerator is attached to the lid of the machine and holds a polished silver single crystal. This crystal is cooled to $10 \mathrm{~K}$ and serves as a substrate for the silane and d4-silane ices. The silane ices were prepared at $10 \mathrm{~K}$ by depositing silane $(99.99 \%)$ and d4-silane $(99.99 \%)$ at pressures of $6 \times 10^{-8}$ torr for $30 \mathrm{~min}$ onto the cooled silver crystal. The absorptions of the silane and d4-silane samples are compiled in Table 1. To determine the ice thickness quantitatively, we integrated the infrared absorption features at $2163 \mathrm{~cm}^{-1}$ and $876 \mathrm{~cm}^{-1}$ and calculated the ice thickness via the Lambert-Beers relationship (Bennet et al. 2004). Considering the integrated absorption coefficients of these fundamentals, i.e. $2.5 \times 10^{-17} \mathrm{~cm}$ and $2.0 \times 10^{-17} \mathrm{~cm}$, and a density of the silane ice of $0.77 \pm 0.03 \mathrm{~g} \mathrm{~cm}^{-3}$ (Sears \& Morrison 1975), an optical thickness of $0.21 \pm 0.01 \mu \mathrm{m}$ silane can be derived. We can compare our infrared spectra with literature data (Nucara et al. 1997; Calvani et al. 1990; Fournier et al. 1972). This suggests that the frozen high temperature modifications of $\mathrm{SiH}_{4}(\mathrm{I})$ and $\mathrm{SiD}_{4}(\mathrm{I})$ dominate the constitution of the sample. However, the presence of the $v_{2}$ peak, which is infrared-inactive in crystalline $\mathrm{SiH}_{4}(\mathrm{I})$, suggests that the sample is partially disordered, possibly amorphous. We would like to point out that silane is certainly not a perfect matrix material to observe all fundamentals of the newly detected silicon-bearing molecules; smaller fundamentals can be obscured by the absorption of the parent molecules. Nevertheless, the detection of new silicon-containing molecules requires large concentrations of silicon-bearing reactant molecules, which is best achieved in pure silane and perdeutero-silane matrices.

The ices were irradiated at $10 \mathrm{~K}$ with $5 \mathrm{keV}$ electrons generated in an electron gun at beam currents of $10 \mathrm{nA}, 100 \mathrm{nA}$, and $1000 \mathrm{nA}$ by scanning the electron beam over an area of $3.0 \pm 0.4 \mathrm{~cm}^{2}$. We would like to stress that the electron beam is used to crack the silicon-hydrogen bonds - initially in the silane molecule and successively in higher order silicon-bearing molecules. The electron irradiation does not simulate the radiation environment around IRC +10216 . Taking into account the irradiation times of between 10 and $120 \mathrm{~min}$ and the extraction efficiency of $78.8 \%$ of the electrons, this exposes the targets from $3.0 \times 10^{13}$ to $4.5 \times 10^{16}$ electrons. To guarantee an identification of the reaction products 
Table 1. Infrared absorptions of the silane (left column) and d4-silane (center column) frosts (sh: shoulder); $\alpha, \beta$, and $\gamma$ denote lattice modes of the silane sample. The corresponding silane and d4-silane spectra are shown in Fig. 1.

\begin{tabular}{ccc}
\hline \hline Frequency, $\mathrm{cm}^{-1}$ & Frequency, $\mathrm{cm}^{-1}$ & Assignment \\
\hline 4354 & 3183 & $2 v_{3}$ \\
4283 & 3128 & $v_{1}+v_{3}$ \\
$3142(\mathrm{sh})$ & 2264 & $v_{3}+v_{4}+\beta$ \\
3128 & 2243 & $v_{2}+v_{3}$ \\
3087 & 2202 & $v_{3}+v_{4}+\alpha$ \\
3060 & 2173 & $v_{3}+v_{4}$ \\
2300 & 1670 & $v_{3}+\gamma$ \\
2250 & 1638 & $v_{3}+\beta$ \\
2195 & 1607 & $v_{3}+\alpha$ \\
2163 & 1581 & $v_{3}$ \\
1876 & 1359 & $v_{2}+v_{4}+\alpha$ \\
1844 & 1334 & $v_{2}+v_{4}$ \\
1050 & 781 & $v_{2}+\beta / v_{4}+\gamma$ \\
957 & 681 & $v_{2}$ \\
948 & 703 & $v_{4}+\beta$ \\
918 & 681 & $v_{4}+\alpha$ \\
876 & 647 & $v_{4}$ \\
\hline
\end{tabular}

in the ices and those sublimation into the gas phase on line and in situ, a Fourier transform infrared spectrometer (FTIR; solid state) and a quadrupole mass spectrometer (QMS; gas phase) were utilized. The Nicolet 510 DX FTIR spectrometer operates in an absorption-reflection-absorption mode (reflection angle $\left(\alpha=75^{\circ}\right)$ (resolution $0.5-2 \mathrm{~cm}^{-1}$; spectra were averaged for $180 \mathrm{~s}$ ). The infrared beam is coupled via a mirror flipper outside the spectrometer, passes through a differentially pumped potassium bromide $(\mathrm{KBr})$ window, is attenuated in the ice sample before and after reflection at a polished silver waver, and exits the main chamber through a second differentially pumped $\mathrm{KBr}$ window before being monitored via a liquid nitrogen cooled detector. The gas phase is monitored by a quadrupole mass spectrometer (Balzer QMG 420; 1-200 amu mass range) with electron impact ionization of the neutral molecules.

\section{Results}

The response of the irradiation of the silane system at $10 \mathrm{~K}$ is dictated by the initial appearance of the silyl radical, $\mathrm{SiH}_{3}\left(\mathrm{X}^{2} \mathrm{~A}_{1}\right)$. This species could be attributed to the $722 \mathrm{~cm}^{-1}$ band (Table 2, Figs. 1 and 2). The position of this $v_{2}$ umbrella mode agrees well with earlier studies employing hydrogen and noble gas matrices (721-738 $\mathrm{cm}^{-1}$ ) (Andrews \& Wang 2002). A band at $820 \mathrm{~cm}^{-1}$, which was assigned to the $v_{6}$ fundamental of the disilane molecule, $\mathrm{Si}_{2} \mathrm{H}_{6}\left(\mathrm{X}^{1} \mathrm{~A}_{1 \mathrm{~g}}\right.$ ) (Andrews \& Wang 2002), appeared immediately at the beginning of the irradiation (Figs. 1 and 2, Table 3). Note that the less intense deformation mode of the $\mathrm{SiH}_{3}$ group is observable at $935 \mathrm{~cm}^{-1}$ as a shoulder. We would like to stress that the assignments of all peaks were double checked in perdeuterated silane $\left(\mathrm{SiD}_{4}\right)$ matrices.

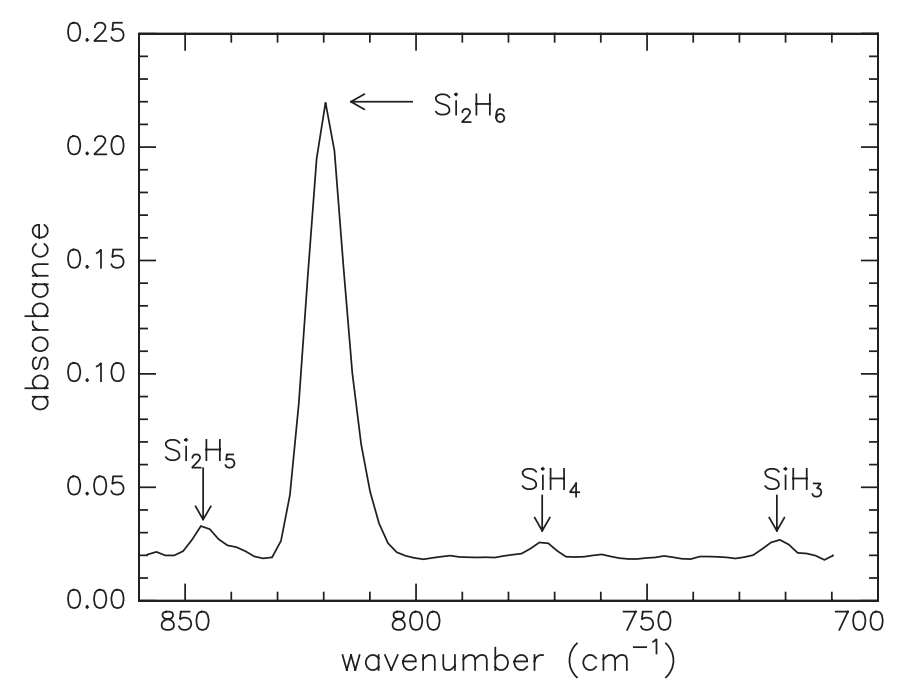

Fig. 1. Absorption of the newly synthesized silicon-bearing silyl radical $\left(\mathrm{SiH}_{3}\right)$ and disilane molecule $\left(\mathrm{Si}_{2} \mathrm{H}_{6}\right)$.
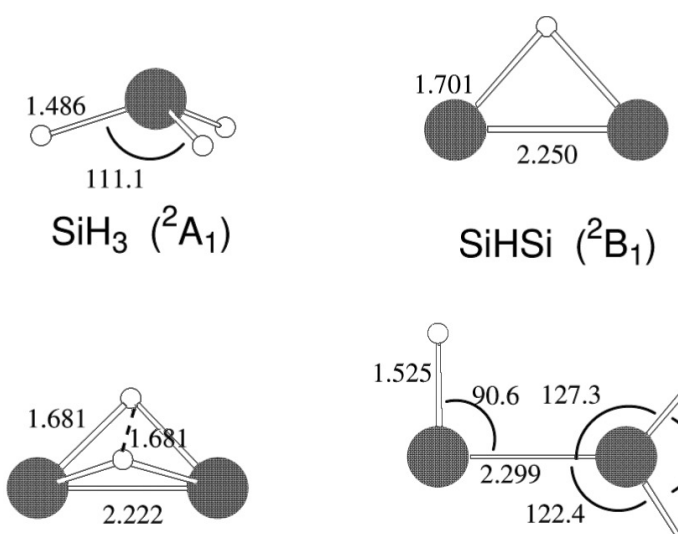

$$
\mathrm{SiH}_{2} \mathrm{Si}\left({ }^{1} \mathrm{~A}_{1}\right)
$$
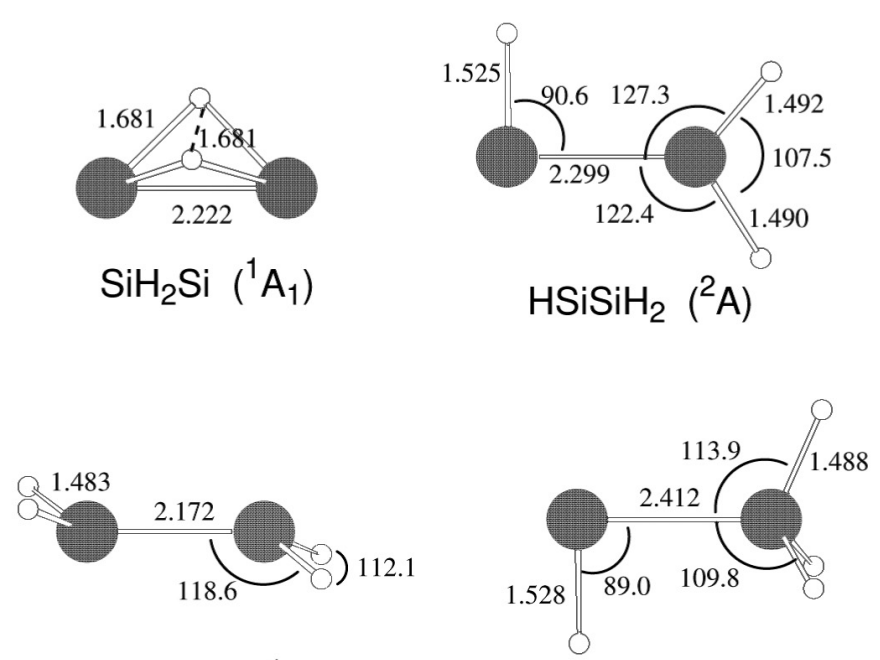

$\mathrm{H}_{2} \mathrm{SiSiH}_{2}\left({ }^{1} \mathrm{~A}_{\mathrm{g}}\right)$

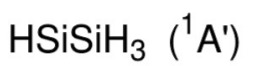

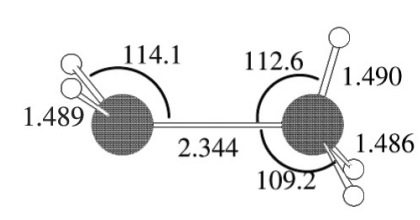

$\mathrm{H}_{2} \mathrm{SiSiH}_{3}\left({ }^{2} \mathrm{~A}^{\prime}\right)$

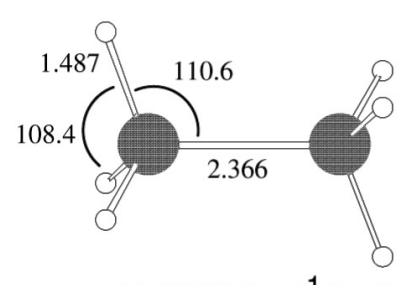

$\mathrm{H}_{3} \mathrm{SiSiH}_{3}\left({ }^{1} \mathrm{~A}_{1 \mathrm{~g}}\right)$
Fig. 2. Calculated structures of various $\mathrm{SiH}_{3}$ and $\mathrm{Si}_{2} \mathrm{H}_{x}(x=1-6)$ species with the B3LYP methods. Bond lengths and bond angles are in $\AA$ and degrees. 
Table 2. Unscaled vibrational frequencies $\left(\mathrm{cm}^{-1}\right)$ and their infrared intensities $\left(\mathrm{km} \mathrm{mol}^{-1}\right)$ of various $\mathrm{SiH}_{n}(n=1-3)$ species calculated with B3LYP/6-311G(d, p) method. The deuterated species are included for completeness.

\begin{tabular}{cccccc}
\hline \hline Mode & Characterization & Frequency & Intensity & Frequency & Intensity \\
\hline & & \multicolumn{2}{c}{$\mathrm{SiH}\left(\mathrm{X}^{2} \Pi\right)$} & \multicolumn{2}{c}{$\mathrm{SiD}\left(\mathrm{X}^{2} \Pi\right)$} \\
$\sigma_{\mathrm{g}}$ & Stretching & 2014 & 331 & 1449 & 171 \\
\hline & & \multicolumn{2}{c}{$\mathrm{SiH}_{2}\left(\mathrm{X}^{1} \mathrm{~A}_{1}\right)$} & \multicolumn{2}{c}{$\mathrm{SiD}_{2}\left(\mathrm{X}^{1} \mathrm{~A}_{1}\right)$} \\
$\mathrm{a}_{1}$ & Symmetric stretching & 2041 & 268 & 1467 & 145 \\
$\mathrm{a}_{1}$ & Bending & 1024 & 93 & 736 & 46 \\
$\mathrm{~b}_{2}$ & Asymmetric stretching & 2039 & 302 & 1468 & 156 \\
\hline & & \multicolumn{2}{c}{$\mathrm{SiH}_{3}\left(\mathrm{X}^{2} \mathrm{~A}_{1}\right)$} & \multicolumn{2}{c}{$\mathrm{SiD}_{3}\left(\mathrm{X}^{2} \mathrm{~A}_{1}\right)$} \\
$\mathrm{a}_{1}$ & Symmetric stretching & 2193 & 5 & 1557 & 3 \\
$\mathrm{a}_{1}$ & Umbrella & 760 & 77 & 561 & 42 \\
$\mathrm{e}$ & Asymmetric stretching & 2234 & 255 & 1616 & 143 \\
$\mathrm{e}$ & Deformation & 937 & 132 & 674 & 67 \\
\hline
\end{tabular}

Here, we detected two fundamentals of the d3-silyl radical, $\mathrm{SiD}_{3}\left(\mathrm{X}^{2} \mathrm{~A}_{1}\right)$, at $669 \mathrm{~cm}^{-1}\left(v_{2}\right)$ and $540 \mathrm{~cm}^{-1}\left(v_{4}\right)$; this assignment corresponds very well with previously assigned peak positions at $668 \mathrm{~cm}^{-1}$ and about $546 \mathrm{~cm}^{-1}$ in noble gas matrices. Likewise, the d6-disilane molecule was detected in our experiments via its absorptions at $606 \mathrm{~cm}^{-1}\left(v_{6}\right)$ and $1541 \mathrm{~cm}^{-1}\left(v_{5}\right)$.

At longer irradiation times, we observed a new absorption at $843 \mathrm{~cm}^{-1}$ in the silane matrix (Fig. 1) and at $621 \mathrm{~cm}^{-1}$ in the perdeutero-silane matrix. We can now compare our calculated absorption frequencies with the experimentally observed ones (Table 3). Accounting for a scaling factor of $0.97-\mathrm{a}$ reasonable value for the $\mathrm{B} 3 \mathrm{LYP} / 6-311 \mathrm{G}(\mathrm{d}, \mathrm{p})$ level of theory the theoretical data predict that the most intense absorption of the disilyl radical (Fig. 2) should be observable at $849 \mathrm{~cm}^{-1}$ $\left(\mathrm{Si}_{2} \mathrm{H}_{5}\left(\mathrm{X}^{2} \mathrm{~A}^{\prime}\right)\right)$ and $621 \mathrm{~cm}^{-1}\left(\mathrm{Si}_{2} \mathrm{D}_{5}\left(\mathrm{X}^{2} \mathrm{~A}^{\prime}\right)\right)$; these predictions are in excellent agreement with our observations. Note that this molecule has not been observed in the reaction of laser ablated silicon atoms in hydrogen matrices (Andrews \& Wang 2002; Wang \& Andrews 2003).

As the exposure time of the silane matrices increases even further, new bands appear at $867 \mathrm{~cm}^{-1}$ (silane matrix; Fig. 3) and $635 \mathrm{~cm}^{-1}$ (d4-silane matrix). A shoulder is also observable at $528 \mathrm{~cm}^{-1}$ in the $\mathrm{d} 4$-silane matrix. We then compared the observed absorptions with those computed for the three energetically most stable $\mathrm{Si}_{2} \mathrm{H}_{4}$ isomers - potential radiolysis products of the disilyl radical (Table 3). Both the $867 \mathrm{~cm}^{-1}$ and $635 \mathrm{~cm}^{-1}$ absorptions can be clearly assigned as the most intense $v_{5}$ fundamental (umbrella mode of the $\mathrm{SiH}_{3} / \mathrm{SiD}_{3}$ group) of the $\mathrm{H}_{3} \mathrm{SiSiH}\left(\mathrm{X}^{1} \mathrm{~A}^{\prime}\right)$ molecule and its d4-isotopomer. Scaling the computed data with a factor of 0.99 gives an excellent agreement with our experimental frequencies. We would like to stress that we were not able to detect any absorption of the thermodynamically more stable $\mathrm{H}_{2} \mathrm{SiSiH}_{2}\left(\mathrm{X}^{1} \mathrm{~A}_{\mathrm{g}}\right.$ ) isomer (Fig. 2). Our calculations suggest that the $\mathrm{H}_{2} \mathrm{SiSiH}_{2}\left(\mathrm{X}^{1} \mathrm{~A}_{\mathrm{g}}\right)$ structure is favorable by about $25 \mathrm{~kJ} \mathrm{~mol}^{-1}\left(27 \mathrm{~kJ} \mathrm{~mol}^{-1}\right.$ is the value with $\mathrm{CCSD}(\mathrm{T})$ method) compared to $\mathrm{H}_{3} \mathrm{SiSiH}\left(\mathrm{X}^{1} \mathrm{~A}^{\prime}\right)$. The reader should note that the most intense $v_{11}$ mode of $\mathrm{H}_{2} \mathrm{SiSiH}_{2}\left(\mathrm{X}^{1} \mathrm{~A}_{\mathrm{g}}\right)$ falls within the $v_{4}$ and $v_{4}+\alpha$ absorptions of the silane matrix (Table 1). We would like to stress that Andrews \& Wang (2002) also identified a $\mathrm{Si}_{2} \mathrm{H}_{4}$ species in hydrogen matrices,

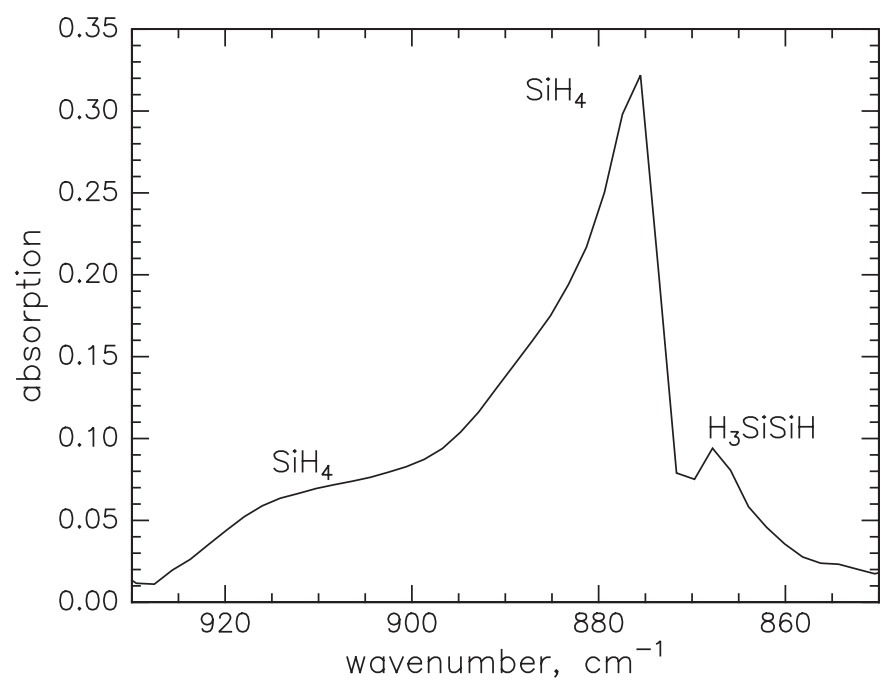

Fig. 3. New absorption features of the silylsilylene $\left(\mathrm{HSiSiH}_{3}, \mathrm{X}^{1} \mathrm{~A}^{\prime}\right)$ isomer at $867 \mathrm{~cm}^{-1}$ in the silane matrix at $10 \mathrm{~K}$; the $v_{4}$ fundamental $\left(876 \mathrm{~cm}^{-1}\right)$ and the $v_{4}+\alpha$ combination bands $\left(918 \mathrm{~cm}^{-1}\right)$ of the silane matrix are also shown.

but no compelling assignment of the isomer was presented. Comparing their data of $861.4 \mathrm{~cm}^{-1}$ (hydrogen in neon) and $634.2 \mathrm{~cm}^{-1}$ (deuterium in neon), we suggest that they observed the $\mathrm{H}_{3} \mathrm{SiSiH}\left(\mathrm{X}^{1} \mathrm{~A}^{\prime}\right)$ structure. We would like to point out that Maier et al. (2002) observed this isomer, too. Their strongest absorptions at $860.6 \mathrm{~cm}^{-1}$ and $642.4 \mathrm{~cm}^{-1}$ are very close to our experimentally observed value of $867 \mathrm{~cm}^{-1}$ and $635 \mathrm{~cm}^{-1}$. The less intense peaks are hidden by the silane matrices.

With an enhanced electron exposure, additional bands at $651 \mathrm{~cm}^{-1}$ (Fig. 4; silane target) as well as $493 \mathrm{~cm}^{-1}$ and $683 \mathrm{~cm}^{-1}$ (d4-silane) appeared. These lines cannot be attributed to any $\mathrm{Si}_{2} \mathrm{H}_{x} / \mathrm{Si}_{2} \mathrm{D}_{x}(x=4-6)$ isomer. We matched these data with the energetically most stable $\mathrm{Si}_{2} \mathrm{H}_{3} / \mathrm{Si}_{2} \mathrm{D}_{3}$ structures suggesting that the newly observed bands originate from an electron-induced decomposition of the $\mathrm{Si}_{2} \mathrm{H}_{4} / \mathrm{Si}_{2} \mathrm{D}_{4}$ isomers. Indeed, we verified the existence of the $\mathrm{H}_{2} \mathrm{SiSiH}\left(\mathrm{X}^{2} \mathrm{~A}\right)$ isomer and also its perdeuterated isotopomers via the $v_{5}$ (rocking) and $v_{4}$ (scissoring of $\mathrm{H}_{2} \mathrm{Si}$ part; shoulder) fundamentals. 
Table 3. Unscaled vibrational frequencies $\left(\mathrm{cm}^{-1}\right)$ and their infrared intensities $\left(\mathrm{km} \mathrm{mol}^{-1}\right)$ of $\mathrm{Si}_{2} \mathrm{H}_{x}$ and $\mathrm{Si}_{2} \mathrm{D}_{x}$ species $(x=1-6)$ calculated with B3LYP/6-311G(d, p) method.

\begin{tabular}{|c|c|c|c|c|c|}
\hline Mode & Characterization & Frequency & $\overline{\text { Intensity }}$ & Frequency & $\overline{\text { Intensity }}$ \\
\hline & & \multicolumn{2}{|c|}{$\mathrm{H}_{3} \mathrm{SiSiH}_{3}$} & \multicolumn{2}{|c|}{$\mathrm{D}_{3} \mathrm{SiSiD}_{3}$} \\
\hline$v_{1}\left(\mathrm{a}_{1 \mathrm{~g}}\right)$ & Symmetric stretch & 2219 & 0 & 1580 & 0 \\
\hline$v_{2}\left(\mathrm{a}_{1 \mathrm{~g}}\right)$ & Deformation & 927 & 0 & 694 & 0 \\
\hline$v_{3}\left(\mathrm{a}_{1 \mathrm{~g}}\right)$ & Si-Si stretching & 421 & 0 & 396 & 0 \\
\hline$v_{4}\left(\mathrm{a}_{1 \mathrm{u}}\right)$ & Torsion & 135 & 0 & 96 & 0 \\
\hline$v_{5}\left(\mathrm{a}_{2 \mathrm{u}}\right)$ & Symmetric stretch & 2210 & 122 & 1572 & 71 \\
\hline$v_{6}\left(\mathrm{a}_{2 \mathrm{u}}\right)$ & Deformation & 854 & 546 & 630 & 295 \\
\hline$v_{7}\left(\mathrm{e}_{\mathrm{g}}\right)$ & Stretching & 2220 & 0 & 1604 & 0 \\
\hline$v_{8}\left(\mathrm{e}_{\mathrm{g}}\right)$ & Deformation & 945 & 0 & 675 & 0 \\
\hline$v_{9}\left(\mathrm{e}_{\mathrm{g}}\right)$ & Rocking & 636 & 0 & 484 & 0 \\
\hline$v_{10}\left(\mathrm{e}_{\mathrm{u}}\right)$ & Stretching & 2230 & 208 & 1612 & 118 \\
\hline$v_{11}\left(\mathrm{e}_{\mathrm{u}}\right)$ & Deformation & 960 & 92 & 689 & 48 \\
\hline \multirow[t]{2}{*}{$v_{12}\left(\mathrm{e}_{\mathrm{u}}\right)$} & Rocking & 381 & 27 & 272 & 13 \\
\hline & & \multicolumn{2}{|c|}{$\mathrm{H}_{3} \mathrm{SiSiH}_{2}$} & \multicolumn{2}{|c|}{$\mathrm{D}_{3} \mathrm{SiSiD}_{2}$} \\
\hline$v_{1}\left(\mathrm{a}^{\prime}\right)$ & $\mathrm{SiH}_{3}$ antisymmetric stretch & 2223 & 75 & 1600 & 56 \\
\hline$v_{2}\left(\mathrm{a}^{\prime}\right)$ & Symmetric stretch & 2199 & 72 & 1571 & 15 \\
\hline$v_{3}\left(\mathrm{a}^{\prime}\right)$ & Symmetric stretch & 2188 & 101 & 1564 & 70 \\
\hline$v_{4}\left(\mathrm{a}^{\prime}\right)$ & Umbrella & 949 & 63 & 691 & 20 \\
\hline$v_{5}\left(\mathrm{a}^{\prime}\right)$ & Bending & 936 & 2 & 676 & 16 \\
\hline$v_{6}\left(\mathrm{a}^{\prime}\right)$ & Umbrella & 875 & 420 & 641 & 223 \\
\hline$v_{7}\left(\mathrm{a}^{\prime}\right)$ & Rocking & 596 & 18 & 454 & 10 \\
\hline$v_{8}\left(\mathrm{a}^{\prime}\right)$ & Si-Si stretching & 424 & 3 & 403 & 0 \\
\hline$v_{9}\left(\mathrm{a}^{\prime}\right)$ & Rocking & 405 & 20 & 294 & 11 \\
\hline$v_{10}(\mathrm{a} ")$ & $\mathrm{SiH}_{3}$ antisymmetric stretching & 2231 & 158 & 1613 & 93 \\
\hline$v_{11}(\mathrm{a} ")$ & $\mathrm{SiH}_{2}$ antisymmetric stretching & 2213 & 48 & 1600 & 20 \\
\hline$v_{12}(\mathrm{a} ")$ & Deformation & 950 & 44 & 680 & 23 \\
\hline$v_{13}(\mathrm{a} ")$ & Deformation & 637 & 1 & 481 & 0 \\
\hline$v_{14}(\mathrm{a} ")$ & Deformation & 390 & 21 & 278 & 10 \\
\hline \multirow[t]{2}{*}{$v_{15}(\mathrm{a} ")$} & Torsion & 127 & 0 & 91 & 0 \\
\hline & & \multicolumn{2}{|c|}{$\mathrm{H}_{2} \mathrm{SiSiH}_{2}$} & \multicolumn{2}{|c|}{$\mathrm{D}_{2} \mathrm{SiSiD}_{2}$} \\
\hline$v_{1}\left(\mathrm{a}_{\mathrm{g}}\right)$ & Symmetric $\mathrm{SiH}$ stretch & 2229 & 0 & 1596 & 0 \\
\hline$v_{2}\left(\mathrm{a}_{\mathrm{g}}\right)$ & Symmetric $\mathrm{SiH}_{2}$ bend & 956 & 0 & 713 & 0 \\
\hline$v_{3}\left(\mathrm{a}_{\mathrm{g}}\right)$ & $\mathrm{Si}-\mathrm{Si}$ stretch & 562 & 0 & 614 & 0 \\
\hline$v_{4}\left(\mathrm{a}_{\mathrm{g}}\right)$ & Rocking & 324 & 0 & 254 & 0 \\
\hline$v_{5}\left(\mathrm{a}_{\mathrm{u}}\right)$ & Asymmetric $\mathrm{SiH}$ stretch & 2258 & 148 & 1634 & 79 \\
\hline$v_{6}\left(\mathrm{a}_{\mathrm{u}}\right)$ & Torsion & 525 & 0 & 371 & 0 \\
\hline$v_{7}\left(\mathrm{a}_{\mathrm{u}}\right)$ & Deformation & 348 & 23 & 249 & 12 \\
\hline$v_{8}\left(\mathrm{~b}_{\mathrm{g}}\right)$ & Asymmetric $\mathrm{SiH}$ stretch & 2246 & 0 & 1625 & 0 \\
\hline$v_{9}\left(\mathrm{~b}_{\mathrm{g}}\right)$ & Deformation & 616 & 0 & 469 & 0 \\
\hline$v_{10}\left(\mathrm{~b}_{\mathrm{u}}\right)$ & Symmetric $\mathrm{SiH}$ stretch & 2225 & 110 & 1590 & 61 \\
\hline$v_{11}\left(\mathrm{~b}_{\mathrm{u}}\right)$ & Symmetric $\mathrm{SiH}_{2}$ bend & 920 & 183 & 665 & 95 \\
\hline$v_{12}\left(\mathrm{~b}_{\mathrm{u}}\right)$ & Rocking & 447 & 32 & 327 & 17 \\
\hline & & & & & \\
\hline$v_{1}\left(\mathrm{a}^{\prime}\right)$ & SiH stretching & 2214 & 128 & 1597 & 75 \\
\hline$v_{2}\left(\mathrm{a}^{\prime}\right)$ & Symmetric $\mathrm{SiH}$ stretching & 2179 & 76 & 1553 & 40 \\
\hline$v_{3}\left(\mathrm{a}^{\prime}\right)$ & $\mathrm{SiH}$ stretching & 2037 & 206 & 1465 & 106 \\
\hline$v_{4}\left(\mathrm{a}^{\prime}\right)$ & $\mathrm{SiH}_{3}$ deformation & 933 & 71 & 671 & 60 \\
\hline$v_{5}\left(\mathrm{a}^{\prime}\right)$ & $\mathrm{SiH}_{3}$ umbrella & 868 & 222 & 641 & 78 \\
\hline$v_{6}\left(\mathrm{a}^{\prime}\right)$ & SiH bending & 716 & 62 & 533 & 42 \\
\hline$v_{7}\left(\mathrm{a}^{\prime}\right)$ & Rocking & 426 & 24 & 377 & 16 \\
\hline$v_{8}\left(\mathrm{a}^{\prime}\right)$ & Si-Si stretching & 368 & 8 & 288 & 9 \\
\hline$v_{9}\left(\mathrm{a}^{\prime \prime}\right)$ & Asymmetric $\mathrm{SiH}$ stretching & 2188 & 109 & 1580 & 63 \\
\hline$v_{10}(\mathrm{a} ")$ & $\mathrm{SiH}_{3}$ deformation & 957 & 38 & 686 & 21 \\
\hline$v_{11}(\mathrm{a})$ & Deformation & 386 & 29 & 280 & 15 \\
\hline$v_{12}(\mathrm{a} ")$ & Torsion & 51 & 10 & 37 & 5 \\
\hline & & & & & \\
\hline$v_{1}(\mathrm{a})$ & Asymmetric Si-H stretch & 2210 & 121 & 1596 & 68 \\
\hline$v_{2}(\mathrm{a})$ & Symmetric Si-H stretch & 2180 & 109 & 1562 & 57 \\
\hline$v_{3}(\mathrm{a})$ & $\mathrm{Si}-\mathrm{H}$ stretch & 2041 & 182 & 1468 & 92 \\
\hline$v_{4}(\mathrm{a})$ & $\mathrm{SiH}_{2}$ scissor & 965 & 93 & 702 & 42 \\
\hline$v_{5}(\mathrm{a})$ & Rocking & 683 & 24 & 511 & 18 \\
\hline$v_{6}(\mathrm{a})$ & Deformation & 448 & 6 & 422 & 7 \\
\hline$v_{7}(\mathrm{a})$ & Out-of-plane & 402 & 9 & 298 & 4 \\
\hline$v_{8}(\mathrm{a})$ & Deformation & 385 & 13 & 279 & 7 \\
\hline$v_{9}(\mathrm{a})$ & Torsion & 192 & 7 & 142 & 4 \\
\hline & & & & & \\
\hline$v_{1}\left(\mathrm{a}_{1}\right)$ & Symmetric H stretch & 1615 & 9 & 1150 & 5 \\
\hline$v_{2}\left(\mathrm{a}_{1}\right)$ & Asymmetric $\mathrm{H}$ stretch & 963 & 54 & 712 & 25 \\
\hline$v_{3}\left(\mathrm{a}_{1}\right)$ & Si-Si stretch & 522 & 1 & 505 & 3 \\
\hline$v_{4}\left(\mathrm{a}_{2}\right)$ & Asymmetric H-shift & 1082 & 0 & 776 & 0 \\
\hline$v_{5}\left(\mathrm{~b}_{1}\right)$ & Asymmtric $\mathrm{H}$ deform & 1533 & 21 & 1096 & 11 \\
\hline$v_{6}\left(\mathrm{~b}_{1}\right)$ & Symmetric H-shift & 1174 & 372 & 852 & 195 \\
\hline & & & & & \\
\hline$v_{1}\left(\mathrm{a}_{1}\right)$ & Sym H stretch & 1476 & 41 & 1057 & 21 \\
\hline$v_{2}\left(\mathrm{a}_{1}\right)$ & $\mathrm{Si}-\mathrm{Si}$ stretch & 509 & 0 & 507 & 0 \\
\hline$v_{3}\left(\mathrm{~b}_{2}\right)$ & H shift & 1035 & 129 & 746 & 67 \\
\hline
\end{tabular}




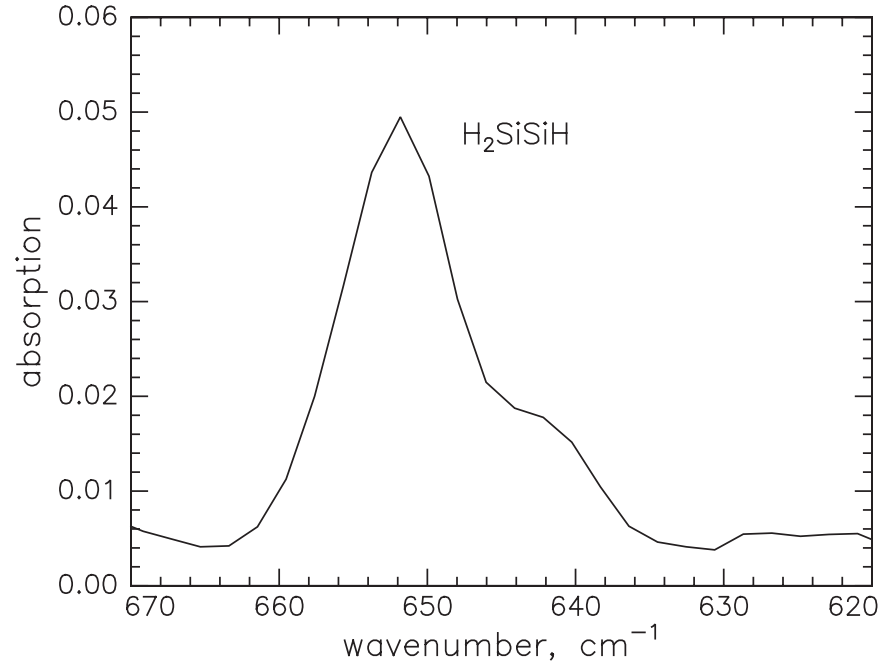

Fig. 4. New absorption features of the silylsilylenyl radical $\left(\mathrm{HSiSiH}_{2}\right.$, $\mathrm{X}^{2} \mathrm{~A}$ ) isomer at $651 \mathrm{~cm}^{-1}$ in the silane matrix at $10 \mathrm{~K}$.

Table 4. Compilation of newly observed hydrogenated silicon-bearing molecules and their absorptions.

\begin{tabular}{lcc}
\hline \hline Species & Frequency, $\mathrm{cm}^{-1}$ & Fundamental \\
\hline $\mathrm{SiH}_{3}$ & 722 & $v_{2}$ \\
$\mathrm{Si}_{2} \mathrm{H}_{6}$ & 820 & $v_{6}$ \\
& 935 & $v_{8}$ \\
$\mathrm{Si}_{2} \mathrm{H}_{5}$ & 843 & $v_{6}$ \\
$\mathrm{H}_{3} \mathrm{SiSiH}$ & 867 & $v_{5}$ \\
$\mathrm{H}_{2} \mathrm{SiSiH}_{2}$ & 898 & $v_{11}$ \\
$\mathrm{H}_{2} \mathrm{SiSiH}$ & 651 & $v_{5}$ \\
\hline
\end{tabular}

A scaling factor of about 0.97 converts the calculated absorptions into the experimentally observed lines. For $\mathrm{H}_{2} \mathrm{SiSiH}\left(\mathrm{X}^{2} \mathrm{~A}\right)$, the more intense $v_{1}-v_{4}$ modes overlap with those of the silane molecule. Compared to the estimation by Sari et al. (2003), which suggested that the bridged $\mathrm{H}_{2} \mathrm{SiHSi}\left(\mathrm{X}^{2} \mathrm{~A}^{\prime \prime}\right)$ isomer is the energetically most stable one followed by $\mathrm{H}_{2} \mathrm{SiSiH}\left(\mathrm{X}^{2} \mathrm{~A}\right)\left(+1 \mathrm{~kJ} \mathrm{~mol}^{-1}\right)$ and $\mathrm{H}_{3} \mathrm{SiSi}\left(\mathrm{X}^{2} \mathrm{~A} "\right)$ $\left(+13 \mathrm{~kJ} \mathrm{~mol}^{-1}\right)$, our calculation leads to the conclusion that all three isomers lie within $0.5 \mathrm{~kJ} \mathrm{~mol}^{-1}$. We were not able to detect any absorption of any $\mathrm{Si}_{2} \mathrm{H}_{2}$ isomers.

After the experiment, the silane sample was heated at a rate of $0.5 \mathrm{~K} \mathrm{~min}^{-1}$ to $293 \mathrm{~K}$. After the sublimation of the silane matrix at $80 \mathrm{~K}$, we observed additional peaks at $898 \mathrm{~cm}^{-1}$ (silane experiment) and $658 \mathrm{~cm}^{-1}$ (d4-silane experiment). These lines were compared with Table 3 . Here, we identified the $898 \mathrm{~cm}^{-1}$ and $658 \mathrm{~cm}^{-1}$ absorptions as the most intense $v_{11}$ (symmetric $\mathrm{SiH}_{2} / \mathrm{SiD}_{2}$ bending) fundamental of the thermodynamically most stable $\mathrm{H}_{2} \mathrm{SiSiH}_{2}\left(\mathrm{X}^{1} \mathrm{~A}_{\mathrm{g}}\right)$ isomer and its perdeuterated counterpart (Fig. 2, Table 4). It is important to compare our findings with an earlier study of Maier et al. (2002). Here, the authors observed the strongest absorption at $904.3 \mathrm{~cm}^{-1}$ close to our observed fundamental at $898 \mathrm{~cm}^{-1}$; two weak silicon-hydrogen stretching modes at $2180.2 \mathrm{~cm}^{-1}$ and $2207.8 \mathrm{~cm}^{-1}$ are obscured in our silane matrix.
Table 5. Calculated dipole moments and rotational constants for $\mathrm{SiH}_{x}(x=1-3)$ and $\mathrm{Si}_{2} \mathrm{H}_{x}(x=1-6)$ species obtained with the B3LYP method.

\begin{tabular}{lcrrr}
\hline \hline & $\begin{array}{c}\text { Dipole moment } \\
\text { (debye) }\end{array}$ & \multicolumn{3}{c}{ Rotational constants } \\
& 0.13 & 0.00 & 220.37 & 220.37 \\
\hline $\mathrm{SiH}$ & 0.15 & 236.51 & 209.18 & 110.99 \\
$\mathrm{SiH}_{2}$ & 0.20 & 140.81 & 140.81 & 83.44 \\
$\mathrm{SiH}_{3}$ & 0.43 & 313.40 & 7.13 & 6.98 \\
$\mathrm{SiHSi}$ & 0.36 & 159.56 & 7.20 & 7.12 \\
$\mathrm{SiH}_{2} \mathrm{Si}_{\mathrm{HiH}}$ & 0.89 & 96.15 & 6.05 & 5.72 \\
$\mathrm{HSiSiH}$ & & 75.38 & 6.34 & 5.93 \\
$\mathrm{H}_{2} \mathrm{SiSiH}_{2}$ & 0 & 62.33 & 5.34 & 5.21 \\
$\mathrm{HSiSiH}_{3}$ & 0.30 & 53.05 & 5.29 & 5.17 \\
$\mathrm{H}_{2} \mathrm{SiSiH}_{3}$ & 0.11 & 43.00 & 5.01 & 5.01 \\
$\mathrm{H}_{3} \mathrm{SiSiH}_{3}$ & 0 & & & \\
\hline
\end{tabular}

\section{Astrophysical implications}

Our combined experimental and theoretical investigations provide reliable infrared absorptions and their intensities of astrophysically important hydrogenated, silicon-bearing molecules (Table 4); all assignments were carefully verified in d4-silane matrices. Two classes of molecules could be identified from the present laboratory study. These are $\mathrm{SiH}_{x}$ species $(x=1-3)$ and $\mathrm{Si}_{2} \mathrm{H}_{x}(x=1-6)$. The $v_{2}$ fundamental of the silyl radical $\left(\mathrm{SiH}_{3} ; 722 \mathrm{~cm}^{-1}\right)$ can be detected easily in future infrared spectroscopic studies of the circumstellar envelope of IRC +10216 . Although photodissociation of silane molecules preferentially yields the $\mathrm{SiH}_{2}\left(\mathrm{X}^{1} \mathrm{~A}_{1}\right)$ and $\mathrm{SiH}\left(\mathrm{X}^{2} \Pi\right)$ species, whose infrared absorptions can be found in Table 2 , reactions of photolytically generated, suprathermal hydrogen atoms with silane can actually yield the silyl radical via a direct abstraction reaction (Eq. (1)):

$\mathrm{SiH}_{4}\left(\mathrm{X}^{1} \mathrm{~A}_{1}\right)+\mathrm{H}\left({ }^{2} \mathrm{~S}_{1 / 2}\right) \longrightarrow \mathrm{SiH}_{3}\left(\mathrm{X}^{2} \mathrm{~A}_{1}\right)+\mathrm{H}_{2}\left(\mathrm{X}^{1} \Sigma_{\mathrm{g}}^{+}\right)$.

Note that the corresponding methyl radical, $\mathrm{CH}_{3}\left(\mathrm{X}^{2} \mathrm{~A}_{1} "\right)$, has been observed in the atmospheres of the giant planets Saturn (Atreya et al. 1999; Bezard et al. 1999) and Neptune (Bezard et al. 1998; Lee et al. 2000) utilizing the Infrared Space Observatory (ISO). Only upper limits have been derived for Jupiter, Uranus, and Titan (Lee et al. 2000; Atreya et al. 2003). The methyl radical has also been detected in the interstellar medium toward the Galactic Center Sagittarius A via the $v_{2}$ Q-branch at $16.5 \mu \mathrm{m}$ and the $\mathrm{R}(0)$ line at $16.0 \mu \mathrm{m}$ with abundances of $1.3 \pm 0.7 \times 10^{-8}$ relative to hydrogen (Feuchthuber et al. 2000), too. However, the silane species and the corresponding silyl radical have eluded a positive identification in the atmospheres of any solar system body so far; only upper limits of silane in Saturn have been published (Larson et al. 1980).

Considering the $\mathrm{Si}_{2} \mathrm{H}_{x}(x=1-6)$ and the homologues $\mathrm{C}_{2} \mathrm{H}_{x}(x=1-6)$ series, we would like to stress that silicon-bearing species have been detected neither in the solar system nor in the interstellar medium. On the other hand, 
members of the homologues $\mathrm{C}_{2} \mathrm{H}_{x}(x=1-6)$ series are ubiquitous in extraterrestrial environments. Here, ethane $\left(\mathrm{C}_{2} \mathrm{H}_{6}\right)$ and acetylene $\left(\mathrm{C}_{2} \mathrm{H}_{2}\right)$ are actually present in the atmospheres of the giant planets Jupiter and Saturn (Fouchet et al. 1999; Courtin et al. 1984) as well as Uranus (Atreya et al. 1999; Bishop et al. 1990) and Neptune (Conrath et al. 1989; Orton et al. 1992). Ethylene $\left(\mathrm{C}_{2} \mathrm{H}_{4}\right)$ was only observed in Jupiter and Neptune (Encrenaz et al. 2002; Fegley 1995). Ethane has also been identified in comets such as 153P/Ikeya-Zhang (Kawakita et al. 2003). Note that ethylene (Goldhaber et al. 1987), acetylene (Cernicharo et al. 1999), and the ethinyl radical (Fuente et al. 1998) have been detected in the circumstellar envelope of IRC +10216 . Our studies provide solid data for a prospective infrared spectroscopic search $\mathrm{Si}_{2} \mathrm{H}_{x}(x=1-6)$, preferentially in the $600-800 \mathrm{~cm}^{-1}$ range (16.6-12.5 $\mu \mathrm{m}$; Tables 3 and 4$)$. These investigations should also help to elucidate the hitherto poorly understood organo-silicon chemistry in circumstellar envelopes of carbon-rich, dying stars.

Acknowledgements. The experiments were supported by the University of Hawai'i at Manoa (R.I.K.), by the Particle Physics and Astronomy Research Council (PPARC, UK) (R.I.K.), and by Osaka Vacuum (Japan) (R.I.K.). The computations were carried out at the Research Center for Computational Science, Japan, and supported by the Grants-in-Aid for Scientific Research on Priority Areas from the Ministry of Education, Science, and Culture, Japan (Y.O.).

\section{References}

Aka, B., \& Boch, E. J. 2002, Photochem. Photobiol. A, 150, 257 Andrews, L., \& Wang, W. 2002, J. Phys. Chem. A, 106, 7696 Apponi, A. J., McCarthy, M. C., Gottlieb, C. A., \& Thaddeus, P. 1999, ApJ, 516, L103

Ashfold, M. N. R., Mordaunt, D. H., \& Wilson, S. H. S. 1996, Adv. Photochem., 21, 217

Atreya, S. K., Edgington, S. G., Encrenaz, Th., \& Feuchtgruber, H. 1999, European Space Agency (Special Publication) SP, 149

Atreya, S. K., Mahaffy, P. R., Niemann, H. B., Wong, M. H., \& Owen, T. C. 2003, Planetary and Space Science, 51, 105

Barratt, A. 1978, ApJ, 220, L81

Becke, A. D. 1993, J. Chem. Phys., 98, 5648

Bennett, C., Jamieson, C., Mebel, A. M., \& Kaiser, R. I. 2004, Phys. Chem. Chem. Phys., 6, 735

Bezard, B., Feuchtgruber, H., Moses, J. I., \& Encrenaz, T. 1998, A\&A, 334, L41

Bezard, B., Romani, P. N., Feuchtgruber, H., \& Encrenaz, T. 1999, ApJ, 515, 868

Bieging, J. H., \& Nguyen, Q. R. 1989, ApJ, 343, L25

Bishop, J., Atreya, S. K., Herbert, F., \& Romani, P. 1990, Icarus, 88, 448

Blanco, A., Birghesi, A., Fonti, S., \& Orofino, V. 1998, A\&A, 330, 505

Borsella, E., \& Fantoni, R. 1988, Chem. Phys. Lett., 150, 542

Calvani, P., Ciotti, C., Cunsolo, S., \& Lupi, S. 1990, Solid State Commun., 75, 189

Cernicharo, J., Gottlieb, C. A., Guelin, M., Thaddeus, P., \& Vrtilek, J. M. 1989, ApJ, 341, L25

Cernicharo, J., Yamamura, I., Gonzalez-Alfonso, E. G., et al. 1999, ApJ, 526, L41

Chandra, S., \& Sahu, A. 1993, A\&A, 272, 700
Cizek, J. 1969, Adv. Chem. Phys., 14, 35

Conrath, B., et al. 1989, Science, 246, 1454

Cook, P. A., Ashfold, M. N. R., Jee, Y-J., et al. 2001, Phys. Chem. Chem. Phys., 3, 1848

Courtin, R., Gautier, D., Marten, A., Bezard, B., \& Hanel, R. 1984, ApJ, 287, 899

Encrenaz, Th. 2002, Adv. Space Res., 30, 1967

Fabian, D., Henning, T., Jager, C., et al. 2001, A\&A, 378, 228

Fegley, B. 1995, in AGU Handbook of Physics Constants, ed. T. Ahrens, 320

Feuchtgruber, H., Helmich, F. P., Van Dishoeck, E. F., \& Wright, C. M. 2000, ApJ, 535, L111

Fouchet, Th., Lellouch, E., Encrenaz, Th., et al. 1999, European Space Agency, 177

Fournier, R. P., The, N. D., Savoie, R., Belzile, R., \& Cabana, A. 1972, Can. J. Chem., 50, 35

Frisch, M. J., et al. 2002, GAUSSIAN98 Revision A11, Gaussian, Inc.: Pittsburgh, P. A.

Fuente, A., Cernicharo, J., \& Omont, A. 1998, A\&A, 330, 232

Gensheimer, P. D., \& Snyder, L. E. 1997, ApJ, 490, 819

Glassgold, A. E., \& Mamon, G. A. 1992, in Chemistry and Spectroscopy of Interstellar Molecules, ed. D. K. Bohme et al. (University of Tokyo Press), 261

Glassgold, A. E., Lucas, R., \& Omont, A. 1986, A\&A, 157, 35

Glenewinkel-Meyer, Th., Bartz, J. A., Thorson, G. M., \& Crim, F. F. 1993, J. Chem. Phys., 99, 5944

Goebel J. H., Cheeseman, P., \& Gerbaullt, F. 1995, ApJ, 246, 246

Goldhaber, D. M., \& Betz, A. L. 1984, ApJ, 279, L55

Goldhaber, D. M., Betz, A. L., \& Ottusch, J. J. 1987, ApJ, 314, 356

Guelin, M., Muller, S., Cernicharo, J., et al. 2000, A\&A, 363, L9

Howe, D. A., \& Millar, T. J. 1990, MNRAS, 244, 444

Hu, S.-W., Wang, Y., Wang, X.-Y., Chu, T.-W., \& Liu, X.-Q. 2003, J. Phys. Chem. A, 107, 2954

Kawakita, H., Watanabe, J., Kinoshita, D., Ishiguro, M., \& Nakamura, R. 2003, ApJ, 590, 573

Kendall, R. A., Dunning, T. H., \& Harrison, R. J. 1992, J. Chem. Phys., 96, 6796

Kraus, G. D., Nuth, J. A., \& Nelson, R. N. 1997, A\&A, 328, 328

Krishnan, R., Binkley, J. S., Seeger, R., \& Pople, J. A. 1980, Chem. Phys., 72650

Langer, W. D., \& Glassgold, A. E. 1990, ApJ, 352, 123

Larson, H. P., Fink, U., Smith, H. A., \& Davis, D. S. 1980, ApJ, 240, 327

Le Picard, S. D., Canosa, A., Pineau des Forets, G., Rebrion-Rowe, C., \& Rowe, B. R. 2001, A\&A, 372, 1064

Ledoux, G., Guillois, O., Huisken, F., et al. 2001, A\&A, 377, 707

Lee, A. Y. T., Yung, Y. L., \& Moses, J. 2000, J. Geophys. Res., 105, 20207

Lee, C., Yang, W., \& Parr, R. G. 1988, Phys. Rev. B, 37, 785

Lloyd Evans, T., Hurst, M. E., \& Sarre, P. J. 2000, MNRAS, 319, 111

Mackay, D. D. S. 1995, Ap\&SS, 224, 507

Maier, G., Reisenauer, H. P., \& Glatthaar, J. 2002, Chem. Eur. J., 8, 4383

McCarthy, M. C., Apponi, A. J., \& Thaddeus, P. 1999, J. Chem. Phys., 110,10645

Nucara, A., Calvani, P., Lupi, S., \& Roy, P. 1997, J. Chem. Phys., 17, 6562

Ohishi, M., Kaifu, N., Kawaguchi, K., et al. 1989, ApJ, 345, L83

Oikawa, S., Tsuda, M., \& Ohtsuka, S. 1994, J. Mol. Structure, 310, 287

Orofino, V., Blanco, A., \& Fonti, S. 1994, A\&A, 282, 657

Orton, G., Lacy, J. H., Achtermann, J. M., Parmar, P., \& Blass, W. E. 1992, Icarus, 100, 541 
Peng, Y., Vogel, S. N., \& Carlstrom, J. E. 1995, ApJ, 455, 223

Perkins, G. G. A., Austin, E. R., \& Lampe, R. W. 1979, J. Am. Chem. Soc., 101, 1109

Pillinger, C. T., \& Russel, S. 1993, J. Chem. Soc. Faraday Trans., 89, 2297

Pople, J. A., Head-Gordon, M., \& Raghavachari, K. J. 1987, Chem. Phys., 87, 5968

Sari, L., McCarthy, M. C., Schaefer III, H. F., \& Thaddeus, P. 2003, J. Am. Chem. Soc., 125, 11409

Sarre, P. J., Hurst, M. E., \& Evans, T. L. 1996, ApJ, 471, L107

Schilke, P., Walmsley, C. M., Pinneau des Forets, G., \& Flower, D. R. 1997, A\&A, 321, 293

Sears, W. M., \& Morrison, J. A. 1975, J. Chem. Phys., 62, 2736

Speck, A. K., Barlow, M. J., \& Skinner, C. J. 1997, MNRAS, 288, 431
Thaddeus, P., Cummins, S. E., \& Linka, R. A. 1984, ApJ, 283, L45

Tonokuna, K., Mo, Y., Matsumi, Y., \& Kawasaki, M. 1992, J. Phys. Chem., 96, 6688

Turner, B. E. 1987, A\&A, 183, L23

Turner, B. E. 1992, ApJ, 388, L35

Walmsley, C. M., Pineau des Forets, G., \& Flower, D. R. 1999, A\&A, 342,542

Wang, X., \& Andrews, L. 2003, J. Am. Chem. Soc., 125, 6581

Wang, Z. X., \& Huang, M. B. 1998, J. Chem. Soc. Faraday Trans., 94, 635

Willacy, K., \& Cherchneff, I. 1998, A\&A, 330, 676

Zavelovich, J., \& Lyman, J. L. 1989, J. Phys. Chem., 93, 5740

Ziurys, L. M., Friberg, P., \& Irvine, W. M. 1989, ApJ, 343, 201 